\title{
Tissue inhibitor of metalloproteinase-2 stimulates mesenchymal growth and regulates epithelial branching during morphogenesis of the rat metanephros
}

\author{
Jonathan Barasch, ${ }^{1}$ Jun Yang, ${ }^{1}$ Jizeng Qiao, ${ }^{1}$ Paul Tempst, ${ }^{2}$ Hediye Erdjument-Bromage, ${ }^{2}$ \\ Winnie Leung, ${ }^{1}$ and Juan A. Oliver ${ }^{1}$
}

${ }^{1}$ Department of Medicine, College of Physicians and Surgeons, Columbia University, New York, New York 10032, USA

${ }^{2}$ Molecular Biology Program, Memorial Sloan-Kettering Cancer Center, New York, New York 10021, USA

Address correspondence to: Jonathan Barasch, Department of Medicine, College of Physicians and Surgeons, Columbia University, New York, New York 10032, USA. Phone: (212) 305-1890, Fax: (212) 305-3475; E-mail: jmb4@columbia.edu.

Received for publication July 14, 1998, and accepted in revised form March 26, 1999.

\begin{abstract}
Development of the embryonic kidney results from reciprocal signaling between the ureteric bud and the metanephric mesenchyme. To identify the signaling molecules, we developed an assay in which metanephric mesenchymes are rescued from apoptosis by factors secreted from ureteric bud cells (UB cells). Purification and sequencing of one such factor identified the tissue inhibitor of metalloproteinase-2 (TIMP-2) as a metanephric mesenchymal growth factor. Growth activity was unlikely due to TIMP-2 inhibition of matrix metalloproteinases because ilomastat, a synthetic inhibitor of these enzymes, had no mesenchymal growth action. TIMP-2 was also involved in morphogenesis of the ureteric bud, inhibiting its branching and changing the deposition of its basement membrane; these effects were due to TIMP-2 inhibition of matrix metalloproteinases, as they were reproduced by ilomastat. Thus, TIMP-2 regulates kidney development by at least 2 distinct mechanisms. In addition, TIMP2 was secreted from UB cells by mesenchymal factors that are essential for ureteric bud development. Hence, the mesenchyme synchronizes its own growth with ureteric morphogenesis by stimulating the secretion of TIMP-2 from the ureteric bud.
\end{abstract}

J. Clin. Invest. 103:1299-1307 (1999).

\section{Introduction}

The adult nephron derives from 2 distinct embryonic tissues, the ureteric bud (a branch of the wolffian duct) and an area of intermediate mesoderm called the metanephric mesenchyme. The ureteric bud invades the metanephric mesenchyme, where it branches, grows, and develops into the collecting duct. Concomitantly, the metanephric mesenchyme grows, converts to epithelia, and develops into the rest of the nephron.

The ureteric bud and metanephric mesenchyme are codependent for survival and development. For example, when metanephric mesenchymal development is defective (1), the ureteric bud fails to grow and branch. Conversely, when ureteric invasion is defective (2-5) or when the ureteric bud is surgically removed, the mesenchyme dies by apoptosis $(6,7)$.

The ureteric bud regulates the development of the metanephric mesenchyme by stimulating both its proliferation and its conversion to epithelia (8), a process that has been termed "induction" of the mesenchyme $(9,10)$. To date, identification of the molecules responsible for this induction has been difficult, primarily because the 2 tissues are codependent for survival. For example, failure of mesenchymal development after deletion of a ureteric molecule does not establish that it is a mesenchymal regulator, because it is possible that the molecule is required for ureteric bud development and that failure of mesenchymal induction is secondary. Furthermore, many molecules active in renal development are either expressed by both mesenchyme and the ureteric bud (e.g., bone morphogenic protein-7; ref. 11) or have receptors in both compartments (e.g., hepatocyte growth factor receptor, c-met; refs. 12, 13). Manipulation of such molecules in the kidney cannot establish whether they directly regulate mesenchymal induction.

To identify molecules that are produced by the ureteric bud and induce the metanephric mesenchyme, we have separated these 2 tissues and have treated isolated mesenchymes with proteins secreted by immortalized ureteric bud cells (UB cells; ref. 8). These cells express many proteins typical of the ureteric bud. More importantly, medium conditioned by UB cells stimulates mesenchymal growth $(8,14)$ and induces mesenchymalto- epithelial conversion (8). By examining the activity of conditioned medium to stimulate growth of isolated metanephric mesenchymes, we have now purified to homogeneity the tissue inhibitor of metalloproteinase-2 (TIMP-2) and identified it as a metanephric mesenchymal growth factor.

We also found that TIMP-2 is secreted from UB cells in response to mesenchymal proteins that regulate ureteric bud development and that TIMP-2 inhibits branching of the ureteric bud. Thus, TIMP-2 has actions in both compartments of the embryonic kidney and is a mediator of the reciprocal interactions that coordinate development of the 2 embryonic tissues fated to become the mature kidney. 


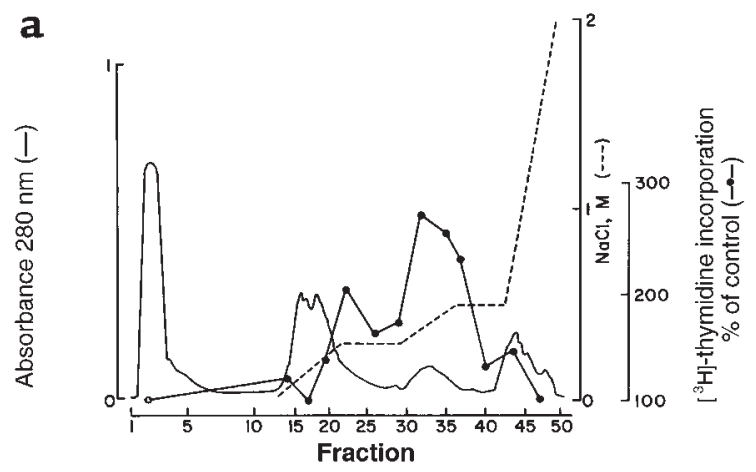

C

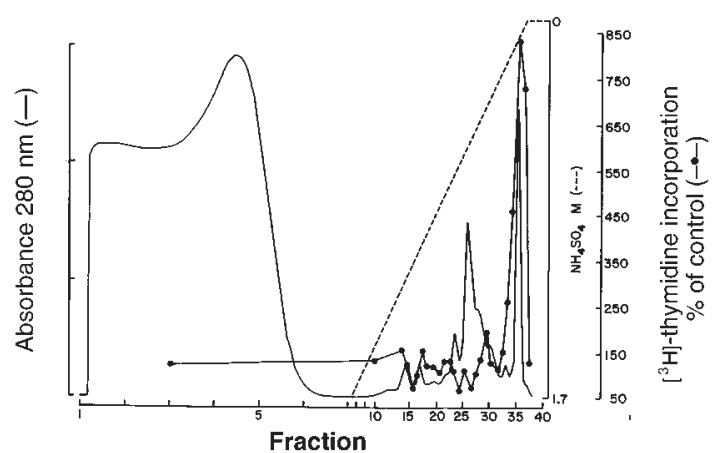

b

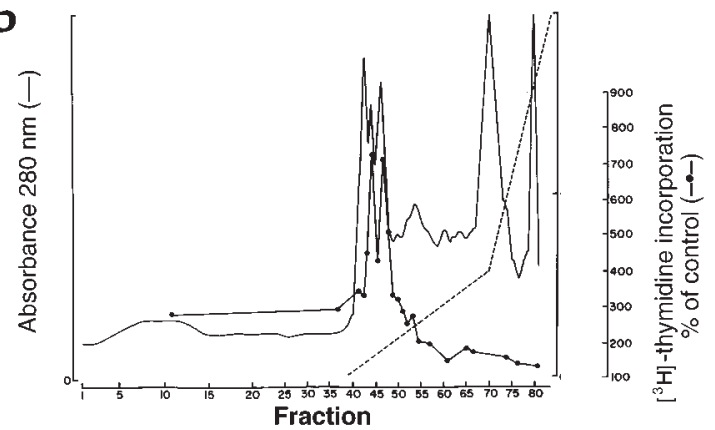

d

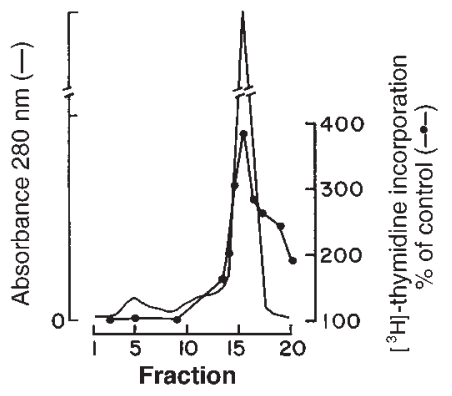

Figure 1

Isolation of TIMP, a metanephric mesenchymal growth factor, from medium conditioned by UB cells. Medium conditioned by UB cells was fractionated by heparin-Sepharose chromatography (a). Three peaks of activity were found (100 $\mu$ g of each fraction was assayed), and fractions $30-40$ were pooled and further purified by a Mono Q anion exchanger (b). Active fractions (fractions 42-48; $10 \mu \mathrm{g}$ of each fraction assayed) were pooled and further purified by phenyl-Sepharose hydrophobic interaction chromatography (c). Activity copurified with a single protein that we purified to homogeneity by Superdex-75 gel filtration $(\mathbf{d})$. In $\mathbf{c}$ and $\mathbf{d}, 10 \%$ volume of each fraction was assayed for $\left[{ }^{3} \mathrm{H}\right]$ thymidine incorporation.

\section{Methods}

Isolation of TIMP-2. UB cells were grown to $90 \%$ confluence in T-150 flasks (Corning-Costar Corp., Acton, Massachusetts, USA) in MEM (GIBCO BRL, Grand Island, New York, USA) with $10 \%$ FCS (HyClone Laboratories, Logan, Utah, USA). The medium was then withdrawn, and the cells were rinsed in MEM and incubated for 24 hours in serum-free MEM. This medium was then discarded, and the cells were cultured in fresh serum-free MEM for 3 days. At the end of 3 days, the medium was collected, centrifuged at $800 \mathrm{~g}$ for 5 minutes, and filtered $(0.22 \mu \mathrm{m})$ to remove debris. Twenty liters of serum-free conditioned medium containing $600 \mathrm{mg}$ of protein was collected in total and concentrated on YM-10 filters (Amicon, Bedford, Massachusetts, USA).

Conditioned medium was fractionated by sequential heparin-Sepharose chromatography (buffer A: $10 \mathrm{mM} \mathrm{NaPO}_{4}$ [pH 7.0]; buffer $\mathrm{B}: 2 \mathrm{M} \mathrm{NaCl}, 10 \mathrm{mM} \mathrm{NaPO}_{4}[\mathrm{pH} 7.0]$ ), anion exchange chromatography (Mono Q; buffer A: bis-Tris [pH 7.2]; buffer B: $1 \mathrm{M} \mathrm{NaCl}$, bis-Tris [pH 7.2]), hydrophobic interaction chromatography (Phenyl-Sepharose PC Smart Column; buffer A: $1.7 \mathrm{M} \mathrm{NH}_{4} \mathrm{SO}_{4}, 50 \mathrm{mM} \mathrm{NaPO}_{4}$ [pH 6.85]; buffer B: $50 \mathrm{mM} \mathrm{NaPO}_{4}$ [pH 6.85]), and gel filtration chromatography (Superdex 75 PC Smart Column; buffer: $150 \mathrm{mM}$ $\mathrm{NaCl}, 50 \mathrm{mM} \mathrm{NaPO} 4$ [pH 7.8], 10\% sucrose). The columns were manufactured by Pharmacia Biotech Inc. (Piscataway, New Jersey, USA).

To locate mesenchymal growth activity, aliquots of every fraction from each column were assayed after washing each 5 times on Microcon-10 filters (Amicon) in PBS [pH 7.4] and then adding the aliquot to 4 metanephric mesenchymes. The mesenchymes were dissected from embryonic day 13 (E13) rat kidneys as described $(8,14)$ and placed on Costar transwell filters (Corning-Costar Corp.) in MEM with 10\% FCS. The best response to fractions of conditioned medium was found using Costar collagen-coated transwell filters (Costar catalog no. 3425; Corning-Costar Corp.). Mesenchymes cultured in MEM with $10 \%$ FCS, without other additions, served as controls. These tissues underwent apoptosis within 12-24 hours of isolation $(8,14)$ and disintegrated and were lost from the filter after 48 hours of culture.

Rescue of mesenchymes from apoptosis was assessed both by $\left[{ }^{3} \mathrm{H}\right]$ thymidine incorporation $(10 \mu \mathrm{Ci} / \mathrm{mL}$ culture medium $)$ and by visual assessment of mesenchymal size at 48 hours of incubation, as described previously $(8,14)$. The visual assessments of mesenchymal survival and $\left[{ }^{3} \mathrm{H}\right]$ thymidine incorporation were always in complete concordance.

The same assay was used to test the effect of a metalloproteinase inhibitor on mesenchymal growth. In 6 independent experiments, 3-4 mesenchymes were treated with $N-[(2 \mathrm{R})-2$ (hydroxamido-carbonylmethyl)-4-methylpentanoyl]-L-tryptophan methylamide (GM6001, or ilomastat; AMS Scientific, Pleasant Hill, California, USA; refs. 15-17) in doses ranging from 0.04 to $25 \mu \mathrm{m}$. $\mathrm{N}$-t-butyloxycarbonyl-L-leucyl-L-tryptophan methylamide (GM1210; AMS Scientific) served as a control for ilomastat. The activity of ilomastat against gelatinases of the metanephric mesenchyme was demonstrated by zymography (18) using commercially prepared gels (Bio-Rad Laboratories Inc., Hercules, California, USA). Each lane of the zymogram contained proteins from 3 metanephric mesenchymes. Ilomastat and GM1210 were dissolved in DMSO. DMSO had no effect on the incorporation of $\left[{ }^{3} \mathrm{H}\right]$ thymidine in isolated metanephric mesenchymes when used at dilutions comparable to those used in treatments with ilomastat.

Identification of TIMP-2. Protein from the gel filtration column was transferred to nitrocellulose paper from a 10\% SDS-PAGE gel. Peptides were generated from the nitrocellulose-bound pro- 


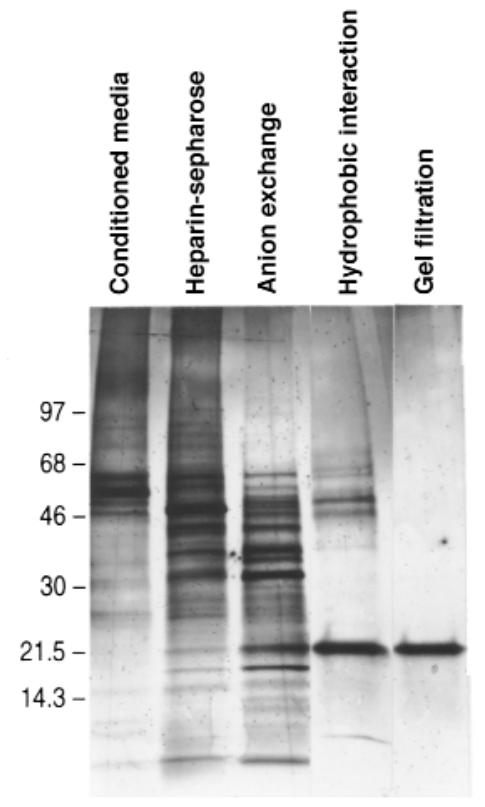

Figure 2

Isolation of TIMP-2. Silver stain of SDS-PAGE gel of medium conditioned by UB cells, and active fractions from the heparin-Sepharose, anion exchange ( $2 \mu \mathrm{g}$ each), hydrophobic, and gel filtration chromatographies ( $5 \%$ volume each).

tein by in situ tryptic digestion (19) and fractionated by reversephase HPLC using a $0.8-\mathrm{mm}$ Vydac C18 column (custom packed by LC-Packings, San Francisco, California, USA; ref. 20). Selected peak fractions were then analyzed by a combination of delayed extraction matrix-assisted laser desorption and ionization reflectron time of flight mass spectrometry (MALDI reTOF MS) (REFLEXIII; Bruker-Daltonik GmbH, Bremen, Germany) and automated Edman sequencing (477A; Applied Biosystems, Foster City, California, USA; refs. 21, 22). Partial peptide sequences were compared with entries in both the nonredundant (nr) and dbEST databases from the National Center for Biotechnology Information using the BLAST program (23). When matches with TIMP-2 were found, mass analysis of several more peptides was done and the results compared with the published sequence by mass-fitting (using PeptideSearch software developed by Matthias Mann, European Molecular Biology Laboratory, Heidelberg, Germany).

RT-PCR for TIMP-2. Poly(A) RNA was prepared from 500 ureteric buds and 200 isolated mesenchymes using RNAzol B (Tel-Test Inc., Friendswood, Texas, USA) followed by selection of poly(A) RNA with oligo(dT) beads (Oligo-Tex; QIAGEN Inc., Santa Clarita, California, USA). RNA was reverse transcribed with a GeneAmp kit (Perkin-Elmer, Foster City, California, USA) in Hot Start tubes (MßP, San Diego, California, USA) for 35 cycles. Primers for rat TIMP-2 were selected using the Prime program (Genetics Computer Group, University of Wisconsin, Madison, Wisconsin, USA). Primer 1 product was $352 \mathrm{bp}$, forward: 5'-AAAGCAATGAGCGAGAAGGAG; reverse: 5'-AGCATGGgatCATAGgGCAG. Primer 2 product was $348 \mathrm{bp}$, forward: 5'-CAAAGGACCTGACAAGGAC; reverse: 5'-TTGATGCAGGCAAAGAAC. Products of each primer set were sequenced.

Immunocytochemistry. E13 kidneys were grown in culture for 2 days on transwell filters (Costar catalog no. 3493; CorningCostar Corp.) and then fixed in $4 \%$ paraformaldehyde, rinsed with $50 \mathrm{mM} \mathrm{NH}_{4} \mathrm{Cl}$, and permeabilized with saponin (0.075\%) for 5 minutes. Tissues were blocked with 10\% FCS (a sample without TIMP) and stained with rabbit anti-rat TIMP-2
(BioSource International, Camarillo, California, USA) at 20 $\mu \mathrm{g} / \mathrm{mL}$. For detection of Dolichos biflorus binding sites, the same procedure was followed, except that gelatin (1\%) served as the blocking reagent and staining with lectin $(40 \mu \mathrm{g} / \mathrm{mL}$; Vector Laboratories, Burlingame, California, USA) was performed at $37^{\circ} \mathrm{C}$ for 30 minutes. For immunodetection of collagen IV, tissues were fixed in methanol $\left(-20^{\circ} \mathrm{C}\right)$ for 5 minutes and stained with rabbit anti-mouse collagen IV (1:2,000; BIODESIGN International, Kennebunk, Maine, USA) using $10 \%$ FCS for blocking.

For immuno-electron microscopy (immuno-EM) of TIMP-2, freshly dissected E14 kidneys were fixed in $4 \%$ paraformaldehyde, $0.2 \%$ glutaraldehyde, $20 \mathrm{mM} \mathrm{NaPO}_{4}$ [pH 7.0], washed with $\mathrm{NH}_{4} \mathrm{Cl}$, and embedded in LR White (Earnest F. Fullam, Inc., Schenectady, New York, USA). Thick sections of the kidneys were first made to find complete, T-shaped ureteric bud branches. To define the tip of a ureteric branch, tissues were sectioned until the presumptive tip just began to recede. The subsequent thin section was then stained for TIMP-2 as described above. Controls included nonimmune rabbit sera instead of anti-TIMP-2, using secondary antibodies only, and incubating EM grids with excess TIMP-2 that competed for staining. Gold particles were counted along the basal surface of the ureteric buds per linear micrometer.

Secretion of TIMP- 2 by UB cells. UB cells $\left(5 \times 10^{4}\right)$ were plated in 6-well plates (Costar catalog no. 3516; Corning-Costar Corp.) and grown for 4 days. Cells were then washed 3 times in serum-free MEM and treated at $37^{\circ} \mathrm{C}$ or $4^{\circ} \mathrm{C}$ with fibroblast growth factor-1 (FGF-1), glial cell-derived neurotrophic factor (GDNF), and FGF-7 (100 ng/mL; R\&D Systems Inc., Minneapolis, Minnesota, USA), or with MEM only. Equal volumes of medium were then subjected to reverse zymography (24), and the amount of TIMP-2 present in the medium from 1-30 minutes was quantitated by laser densitometry. The experiment was repeated 4 times from new plates of UB cells and the data analyzed by ANOVA.

Other assays. TIMP- 2 was detected in dissected ureteric buds and mesenchymes by reverse zymography using $2 \mu \mathrm{g}$ total protein of each sample (24). This assay is approximately $20-50$ times more sensitive to TIMP-2 than to TIMP-1, as judged by loading equal amounts of purified TIMP-1 and TIMP-2 (Boehringer Mannheim Biochemicals, Indianapolis, Indiana, USA; ref. 24). Recombinant TIMP-2 (rTIMP-2) was obtained from Calbiochem-Novabiochem Corp. (San Diego, California, USA) and was purified by sequential immunoaffinity, gel filtration, and anion exchange chromatography according to the manufacturer's instructions. A few experiments were also done with TIMP-2 (Boehringer Mannheim Biochemicals), which was isolated from a melanoma cell line. These 2 commercial preparations of TIMP-2 had identical actions to TIMP-2 isolated from UB cells. The TIMP- 2 concentration in all preparations was assessed by reverse zymography. The rTIMP-2 was denatured by reduction and alkylation as described $(25,26)$.

GDNF was detected in 30 E13 mesenchymes treated with rTIMP-2 $(2 \mu \mathrm{g} / \mathrm{mL})$ for 2 days, using immunoblots with antiGDNF antibodies (R\&D Systems Inc.).

\section{Results}

Identification of TIMP-2, a ureteric protein that rescues metanephric mesenchymes from apoptosis. In tissue culture medium, isolated rat metanephric mesenchymes of E13 rats initiate apoptosis within the first 24 hours $(6,7)$ and involute over the following 24 hours. In contrast, treatment of mesenchymes with medium conditioned by UB cells induces visually obvious growth of the tissue and enhances $\left[{ }^{3} \mathrm{H}\right]$ thymidine incorporation $(8,14)$. 

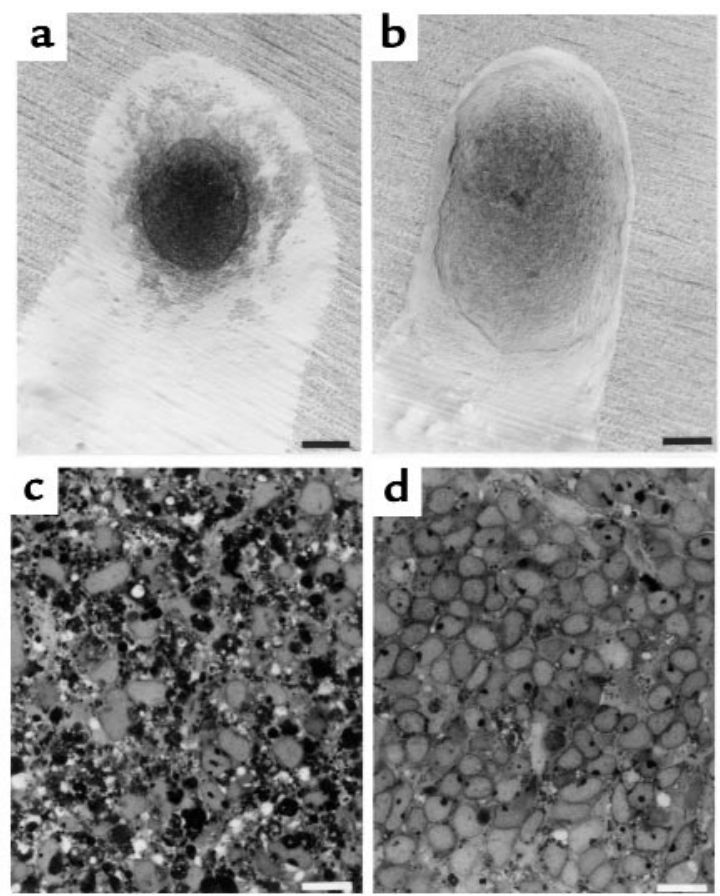

Figure 3

Rescue of isolated E13 metanephric mesenchymes from apoptosis by TIMP-2. Metanephric mesenchyme involutes (a) and is replaced by apoptotic bodies (c) when cultured for 48 hours in tissue culture medium (MEM with $10 \%$ FCS). In contrast, the addition of rTIMP-2 $(2 \mu \mathrm{g} / \mathrm{mL}$ ) rescues metanephric mesenchymal cells from apoptosis (b). Compared with mesenchymes incubated in basal medium, few apoptotic bodies are found when rTIMP-2 is included (d). Scale bars in $\mathbf{a}$ and $\mathbf{b}: 125 \mu \mathrm{m}$. Scale bars in $\mathbf{c}$ and $\mathbf{d}: 10 \mu \mathrm{m}$
Twenty liters of medium conditioned by UB cells (600 mg of protein) was fractionated by heparin-Sepharose chromatography. Both $\left[{ }^{3} \mathrm{H}\right]$ thymidine incorporation and the visual survival of isolated mesenchymes identified 3 separated peaks of activity (Figure 1a). The most active of these peaks (eluting at $0.5 \mathrm{M} \mathrm{NaCl}$ ) was further fractionated by anion exchange (Figure 1b), hydrophobic interaction (Figure 1c), and gel filtration chromatographs (Figure $1 \mathrm{~d}$ ). This yielded a single $23-\mathrm{kDa}$ protein (Figure 2 ) that stimulated $\left[{ }^{3} \mathrm{H}\right]$ thymidine incorporation and rescued isolated metanephric mesenchymes from apoptosis (Figure 3).

Mass spectroscopic analysis and sequencing of tryptic peptides identified the protein as TIMP-2, a protease inhibitor. We confirmed that TIMP-2 is able to rescue isolated metanephric mesenchymes from apoptosis by using 2 additional preparations of TIMP-2 (see Methods). In these experiments, the $\mathrm{ED}_{50}$ of rTIMP-2 was $2 \mathrm{nM}(n=5)$. Saturating concentrations of either rTIMP-2 (40 nM) or TIMP-2 isolated from UB cells stimulated $\left[{ }^{3} \mathrm{H}\right]$ thymidine incorporation 2- to 4-fold. The growth activity of rTIMP-2 was abolished by denaturing the protein. Rescue of mesenchymes from apoptosis was also stimulated by rTIMP-1 (not shown). These data demonstrate that TIMP proteins are able to rescue metanephric mesenchymes from apoptosis and are in agreement with prior demonstrations that TIMP proteins stimulate growth of a variety of cell lines $(17,25,27-30)$.

To determine whether TIMP-2 stimulated mesenchymal growth as a result of its antiprotease activity, we examined the effect of ilomastat (GM6001; refs. 15-17), a matrix metalloproteinase inhibitor. First, using zymography, we found that ilomastat (at dosages as low as $0.04 \mu \mathrm{M}$ ) was active against proteases present in the metanephric mesenchyme, whereas a control compound, lacking antiprotease activity, permitted prominent gelatinolytic activity at $64 \mathrm{kDa}$, an activity previously reported in metanephric mesenchymes (31). However, despite its activity against metanephric metalloproteinases, ilomastat did not stimulate $\left[{ }^{3} \mathrm{H}\right]$ thymidine incorporation in isolated mesenchymes (8 individual doses ranging from 0.04 to $25 \mu \mathrm{M}$ were tested; $n=6$ independent
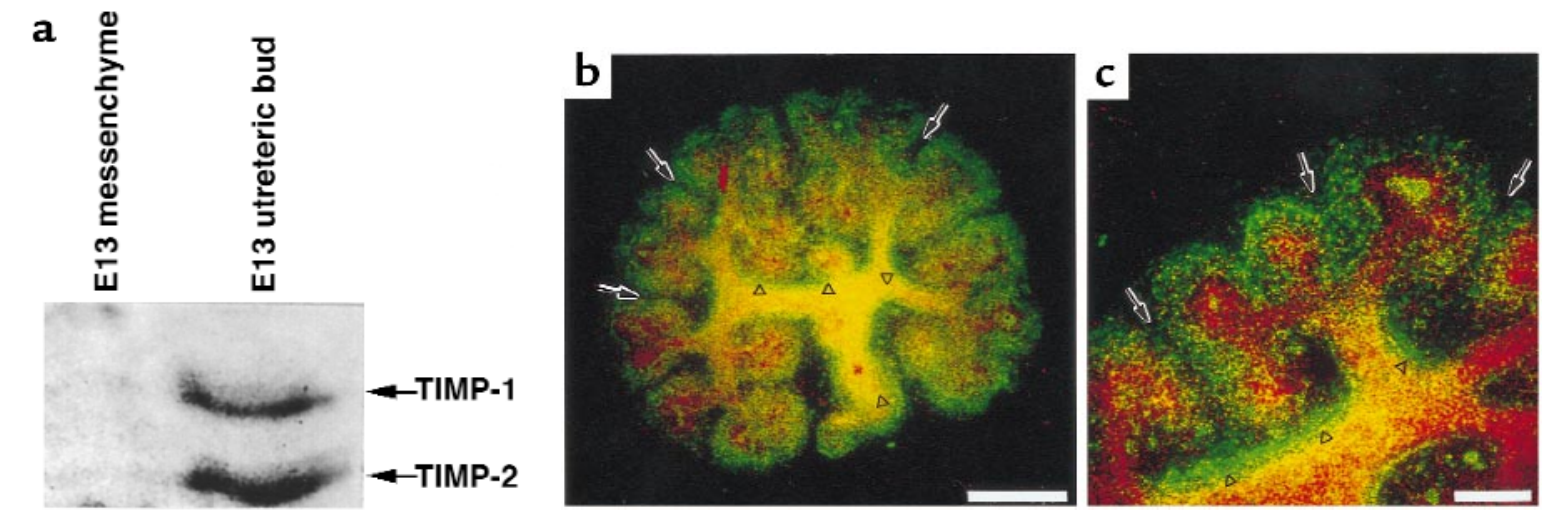

Figure 4

Location of TIMP-2 in embryonic kidney. (a) Reverse zymography of isolated E13 mesenchymes and ureteric buds showing prominent localization of TIMP-2 antiprotease activity in the ureteric compartment. (b and c) Confocal microscopy of TIMP-2 immunofluorescence; a three-dimensional $\mathrm{X}$-Y projection of serial cuts through the kidney. TIMP-2 immunoreactivity (green = fluorescein) is found in the condensed metanephric mesenchyme but not in mesenchymal cells that are more distant from the ureteric bud (arrows). Particularly prominent staining is found in the basement matrix surrounding branches of the ureteric bud (arrowheads), which is defined by binding of $D$. biflorus, a lectin specific for the ureteric bud (red $=$ rhodamine). Scale bar in b: $140 \mu \mathrm{m}$. Scale bar in c: $100 \mu \mathrm{m}$. 

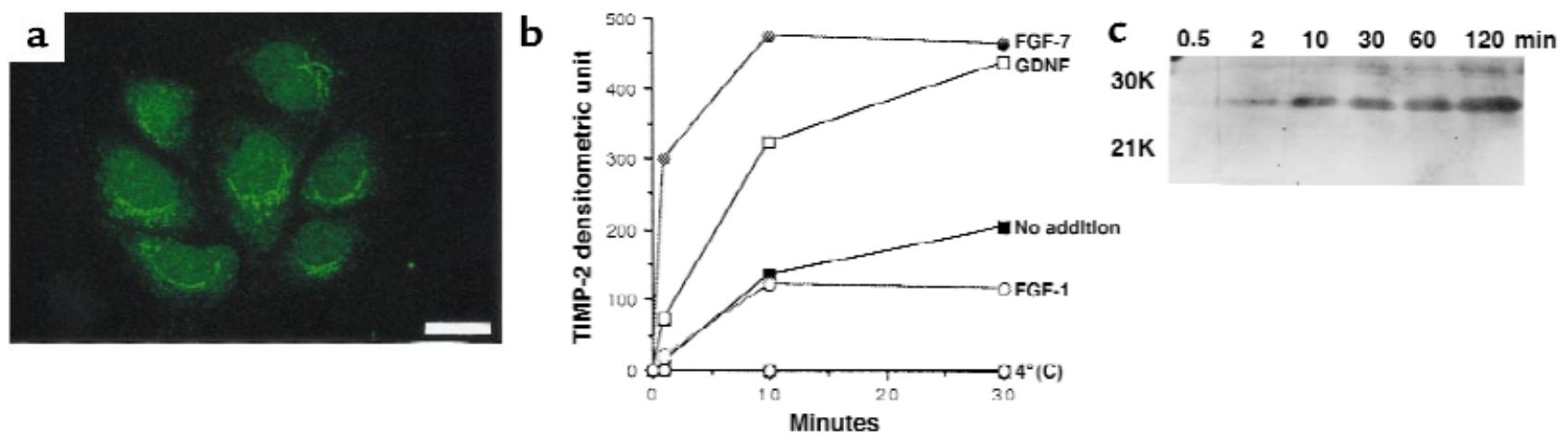

Figure 5

Secretion of TIMP-2 from UB cells is stimulated by mesenchymal proteins that regulate growth and branching of the ureteric bud. (a) UB cells contain immunoreactive TIMP-2. (b) Constitutive secretion of TIMP-2 from UB cells in culture. UB cells were incubated in serum-free MEM, and aliquots collected at the indicated times were assayed by reverse zymography for TIMP-2. (c) Secretion of TIMP-2 is stimulated by GDNF and FGF-7 (100 $\mathrm{ng} / \mathrm{mL} ; P<0.02)$ but not by FGF-1 $(100 \mathrm{ng} / \mathrm{mL})$. At $4^{\circ} \mathrm{C}$, both basal and stimulated secretion of TIMP-2 are abolished. Quantification of TIMP-2 secretion was derived from laser densitometry of reverse zymograms. The curves are averaged from 4 independent experiments and analyzed by ANOVA. Scale bar in a: $100 \mu \mathrm{m}$.

assays). These data indicate that an inhibitor of many matrix metalloproteinases (15), including metanephric proteases, has no growth-promoting activity in metanephric mesenchyme. We conclude that while some of TIMP-2's mesenchymal growth activity may be due to matrix metalloproteinase inhibition, other mechanisms of growth stimulation are likely to be operative.

TIMP-2 in embryonic kidney. Because UB cells are immortalized by SV40 T antigen and may express proteins not normally found in the native ureteric bud, we sought to confirm that TIMP-2 is expressed by the ureteric bud during its invasion of the metanephric mesenchyme. Using RT-PCR in dissected ureteric buds and mesenchymes, we found that both mesenchyme and ureteric bud synthesized TIMP-2 (not shown).

To localize matrix metalloproteinases inhibitor activity of TIMP-2 in the developing kidney, we performed reverse zymography using isolated E13 mesenchymes and ureteric buds (Figure 4a). The assay demonstrated that despite the synthesis of TIMP-2 in both the ureteric bud and the metanephric mesenchyme, all detectable TIMP-2 (and TIMP-1) activity was associated with the ureteric compartment when the kidney initially forms at E13. These data demonstrate that TIMP-2 is produced and likely sequestered by the ureteric bud in vivo.

We further localized TIMP-2 using immunocytochemistry in embryonic kidneys. Consistent with the reverse zymogram, the most intense staining was in the ureteric bud, particularly at branches and clefts (Figure 4, b and c; see Figure 9a). This is consistent with recently published immunolocalizations of TIMP-2 in the embryonic kidney (32). In the metanephric mesenchyme, staining was evident in cells that surround the tips of the ureteric bud, an area of intense proliferation (33) called the condensed mesenchyme. Mesenchymal cells more distal from the ureteric bud were negative (arrows in Figure 4, $\mathrm{b}$ and $\mathrm{c}$ ). These results indicate that TIMP-2 may be a regulator of mesenchymal proliferation and ureteric branching in vivo. Because TIMP- 1 can also rescue metanephric mesenchymes from apoptosis, it may have similar activities as TIMP-2, although the expression and distribution of TIMP-1 in the embryonic kidney is not yet established. Secretion of TIMP-2 from UB cells is regulated by mesenchymal proteins that regulate development of the ureteric bud. Metanephric mesenchymal growth is synchronous with UB invasion, and, as noted above, proliferating condensed mesenchymes that are rich in TIMP- 2 surround each tip of the invading ureteric bud. This suggests that TIMP-2's mesenchymal growth activity may be a mechanism linking ureteric invasion and metanephric growth. To test this, we examined whether mesenchymal factors that control ureteric development could also induce the ureteric bud to secrete TIMP-2. UB cells (Figure 5a) and ureteric buds (not shown) show intracellular storage as well as constitutive secretion of TIMP-2 (Figure 5b). GDNF (34), FGF-7 (35), and FGF-1 (14) are produced by the metanephric mesenchyme, bind to receptors in the ureteric bud as well as in UB cells, and regulate ureteric growth and branching $(2-5,35-39)$.

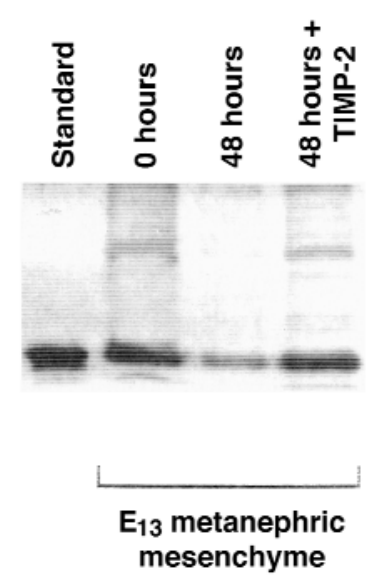

\section{Figure 6}

GDNF in metanephric mesenchymal cells that were rescued from apoptosis by incubation with TIMP-2. GDNF was detected by immunoblots in 6 freshly isolated E13 mesenchymes ( 0 hours) and in 6 mesenchymes maintained in culture by rTIMP-2 for 48 hours ( 48 hours + TIMP-2). In contrast, little reactivity remains in 6 untreated mesenchymes ( 48 hours). Standard is $25 \mathrm{ng}$ of recombinant rat GDNF. 


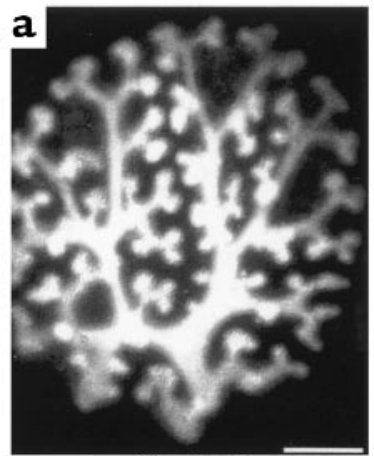

Control

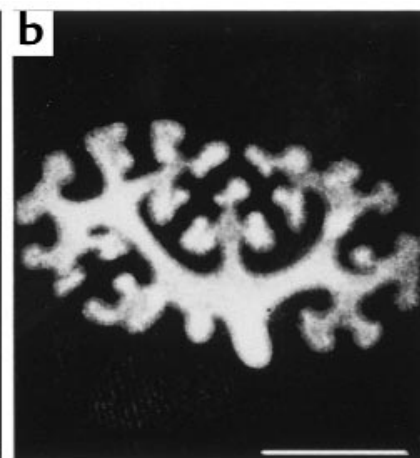

TIMP-2

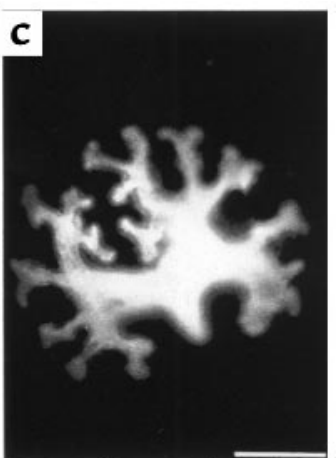

llomastat

\section{Figure 7}

TIMP-2 inhibits ureteric branching in vitro. E13 kidneys were cultured for 4 days with $\mathrm{rTIMP}-2(2 \mu \mathrm{g} / \mathrm{mL})$, and the ureteric bud was then viewed with $D$. biflorus lectin. Treatment with rTIMP-2 inhibits branching of the ureteric bud (b), as compared with kidneys cultured in basal medium (a). Ilomastat $(2 \mu \mathrm{M})$, an inhibitor of matrix metalloproteinases, also inhibited branching of the ureteric bud. Images are three-dimensional X-Y projections of serial confocal cuts through the kidney. Scale bars: $300 \mu \mathrm{m}$.

When monolayers of UB cells were challenged with each of these mesenchymal factors, GDNF and FGF-7 induced an immediate additional secretion of TIMP-2, with a significant effect occurring by the first sampling point of 1 minute (Figure 5c). In contrast, addition of FGF-1 had no effect. Both the basal and stimulated release of TIMP- 2 were abolished at $4^{\circ} \mathrm{C}$. These data suggest that metanephric mesenchymes can alter their own growth by releasing a secretory pool of TIMP-2 from the ureteric bud. Furthermore, since the secretogogues of TIMP-2 also regulate ureteric bud development, these data provide a mechanism by which development of these 2 tissues can be synchronized.

TIMP-2 maintains GDNF-expressing cells. Because secretion of TIMP-2 from UB cells is stimulated by proteins produced in the metanephric mesenchyme, such as GDNF and FGF-7, we examined whether mesenchymal cells expressing GDNF were among those rescued from apoptosis by TIMP-2. As shown in Figure 6, mesenchymes incubated with rTIMP-2 contained readily detectable GDNF, whereas it was only weakly detected in the absence of rTIMP-2, suggesting that TIMP-2 con- tributes to the viability of GDNF-producing cells. The results raise the possibility of a positive-feedback loop between the mesenchyme and ureteric bud that could help synchronize the development of the 2 tissues.

TIMP-2 contributes to ureteric bud morphogenesis. Epithelial morphogenesis in the developing breast, salivary gland, mandible, and kidney is regulated by the anti-matrix metalloproteinase activity of TIMP-1 (16, $31,40-42)$. Moreover, our finding that mesenchymal factors that regulate ureteric development can induce secretion of TIMP-2 suggests that this protein may also be involved in ureteric bud morphogenesis.

To examine the role of TIMP-2 in the development of the ureteric bud, we incubated explanted E13 kidneys with rTIMP-2 and viewed the ureteric bud with the lectin D. biflorus. We found that kidneys cultured with rTIMP$2(2 \mu \mathrm{g} / \mathrm{mL})$ for 4 days had a $43 \%$ reduction in the number of ureteric bud tips compared with control kidneys $(n=23$ for treated and control kidneys, $P<0.02$; Figure 7 , a and b). To determine whether inhibition of ureteric bud branching by TIMP-2 might be due to its anti-matrix metalloproteinase activity, we treated kid-
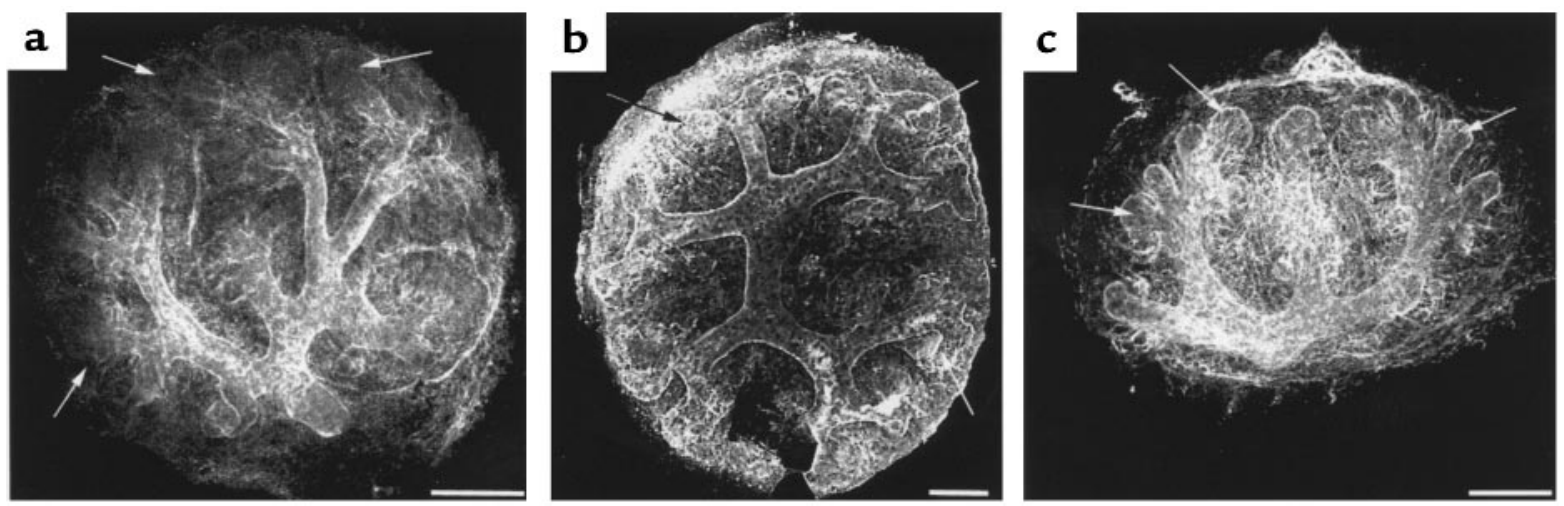

Figure 8

TIMP-2 enhances matrix deposition. E13 kidneys were cultured for 4 days with rTIMP-2 $(2 \mu \mathrm{g} / \mathrm{mL})$ and stained with antibodies to collagen IV. The entire ureteric tree is stained by collagen IV antibodies after rTIMP-2 treatment (b), including tip regions (arrows), while only proximal parts of the ureteric bud are obviously ensheathed by collagen IV in control kidneys (a). A few tips of the ureteric bud are indicated (arrow). (c) Similar to the effect of rTIMP-2, treatment of E13 kidneys with ilomastat $(2 \mu \mathrm{M})$ resulted in matrix deposition throughout the ureteric tree. X-Y projection of serial cuts through the kidney. Scale bars: $100 \mu \mathrm{m}$. 
neys with the matrix metalloproteinase inhibitor ilomastat (15-17). Similar to the effect of TIMP-2, ilomastat $(2 \mu \mathrm{M})$ inhibited branching by $53 \%$ when compared with embryonic kidneys treated with a control reagent for ilomastat, $\quad N$-t-butyloxycarbonyl-L-leucyl-L-tryptophan methylamide, which had no effect $(n=35$ for each group, $P<0.001$; Figure 7c).

We also found that TIMP-2 may alter the composition of the basement membrane of the ureteric bud. Collagen IV immunoreactivity in the basement membrane of the ureteric bud was markedly enhanced in embryonic kidneys treated with rTIMP-2. Furthermore, in these kidneys collagen IV became detectable in the tips of the ureteric bud (Figure 8, a and b), a site normally devoid of basement membrane in vivo $(43,44)$. These effects of TIMP-2 are likely due to its inhibition of metanephric mesenchymal matrix metalloproteinases, because ilomastat also stimulated matrix accumulation (Figure 8c), even at the tips of the branches of the ureteric bud. The control reagent for ilomastat had no effect (not shown).

Finally, by immuno-EM of the ureteric bud in vivo, we found few anti-TIMP-2 gold particles at the tips of the ureteric bud at the ureteric-mesenchymal interface, whereas along branches and in clefts that separate the branches of the bud, there were $2.4 \pm 0.3$ - and $3.7 \pm 0.9$-fold (mean \pm SEM) more anti-TIMP-2 gold particles per micrometer, respectively $(P<0.01$; Figure 9$)$. At these sites, TIMP-2 was abundant in the basement membrane of the ureteric bud. In contrast, at ureteric bud tips there was no basement membrane and little TIMP-2 (Figure 9 and refs. 43, 44).

Taken together, these data suggest that TIMP-2, once secreted from the ureteric bud, associates with its basement membrane where, by inhibiting matrix metalloproteinases, it increases deposition of collagen IV and perhaps other matrix constituents. This could inhibit further branching of the bud and would be consistent with the findings that TIMP can alter matrix composition in the mammary gland (45). Lastly, the data are concordant with the recent finding that GDNF, a secretogogue of TIMP-2, enhances matrix deposition by ureteric buds (46).

\section{Discussion}

When the ureteric bud invades, the metanephric mesenchyme is composed of a few thousand cells. These cells will produce over 10,000 nephrons in a 2-week period, approximately a 1,000-fold increase in cell number (47). In addition, many mesenchymal cells normally undergo apoptosis $(6,7)$, suggesting that even greater degrees of mesenchymal proliferation are required for normal nephron development. This mesenchymal proliferation is critically dependent on signals from the ureteric bud, since removal of the bud from embryonic kidney leads to fulminant mesenchymal apoptosis $(6,8,14)$. To identify these signals, we have developed an in vitro assay that measures the response of isolated metanephric mesenchymes to factors secreted by UB cell lines $(8,14)$.

We now report that UB cells secrete TIMP-2, which rescues isolated metanephric mesenchymes from apoptosis, and that TIMP-2 is produced by the ureteric bud in vivo. In metanephric mesenchyme, we found that TIMP2 is prominently located in the area of proliferating mesenchymal cells that surround the tips of the ureteric bud.
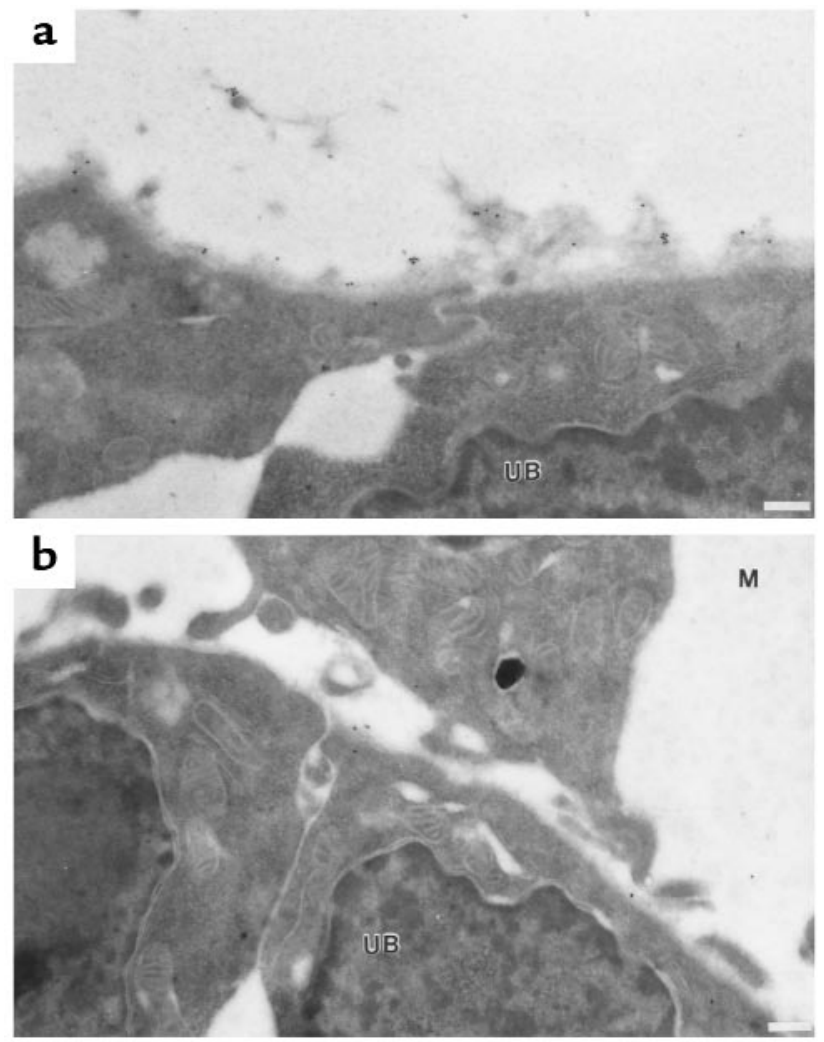

Figure 9

TIMP-2 localizes to the basement membrane of the ureteric bud. (a) Immuno-EM of E14 kidneys showing the localization of TIMP-2 to basement membranes of a cleft between 2 branches of the ureteric tree. (b) In contrast, little TIMP-2 is found at the tips of the ureteric bud, an area devoid of basement membrane. Note the curvature of the basal surface of the epithelia that define the tip of the ureteric bud. $M$, mesenchyme. UB, ureteric bud. Scale bars: $200 \mathrm{~nm}$.

Taken together, these data suggest that TIMP-2 secreted by the ureteric bud is a metanephric mesenchymal growth factor in vivo and that TIMP-2 participates in determining the number of viable mesenchymal cells.

There are several mechanisms by which TIMP-2 could regulate the growth of the mesenchyme. First, TIMP-2 could regulate mesenchymal growth by blocking proteolysis of matrix components important for cell survival - such as entactin in the developing breast (45) and fibronectin in cell cultures (17; see also ref. 48 ) - or by blocking growth factors or growth factor receptors (49). However, in metanephric mesenchyme, $\left[{ }^{3} \mathrm{H}\right]$ thymidine incorporation was not enhanced by ilomastat $(17,30)$, a synthetic matrix metalloproteinase inhibitor. Second, TIMP-2 may activate a unique cell surface receptor that directly stimulates growth. Evidence for this receptor includes binding studies in cultured cells (50), rapid second-messenger activation after the application of TIMP$2(29,51)$, and site-directed mutagenesis that abolished TIMP-1's antiprotease activity but maintained its growth action (52). Thus, while TIMP-2 clearly has anti-matrix metalloproteinase activity in the embryonic kidney, the mechanism of its growth activity is not yet clear. Identification of a unique receptor is needed to definitively establish that TIMP directly stimulates growth. 
The metanephric mesenchyme grows in discrete aggregates (condensed mesenchyme) that surround each tip of the ureteric bud (33). This juxtaposition requires synchronous development of the 2 tissues, believed to be due to a process of reciprocal induction between them. To date, however, no specific reciprocal signaling pathways have been described in the kidney, as they have in the developing limb bud $(53,54)$. We previously found that FGF-2, which is expressed by the ureteric bud and not by mesenchyme, stimulates the growth of mesenchymal cells that, in turn, stimulate branching of the ureteric bud (14). In the present work, we describe a possible mechanism of reciprocal induction whereby mesenchymal proteins (such as FGF-7 and GDNF) that are essential for ureteric development stimulate the secretion of a mesenchymal growth factor (TIMP-2) from ureteric epithelia. Since TIMP-2 is able to promote the survival of at least 1 mesenchymal cell type that signals to the ureteric bud, the findings imply a positive feedback that could maintain mesenchymal signaling of the ureteric bud, and consequently, ureteric signaling of the mesenchyme. We speculate that this type of reciprocal induction could enhance mesenchymal growth at specific sites, such as the condensed mesenchyme (the location of the GDNF-synthesizing cells; ref. 34) that surrounds the tips of the ureteric bud. The recent demonstration that mesenchymal proteoglycans regulate expression of Wnt-11 in the ureteric bud $(33,55)$ may define a third reciprocal loop in the embryonic kidney, because Wnt11 is a secreted protein with likely, but unproven, effects on the metanephric mesenchyme.

In addition to a role in coordinating mesenchymal and ureteric development, our results suggest that TIMP-2 has a direct role in ureteric morphogenesis. First, we found that TIMP-2 is strikingly abundant in nonbranching segments of the ureteric bud, where it locates in the basement membrane. Furthermore, exogenous TIMP-2 increased collagen IV deposition, even at the invading tips, which are normally devoid of basement membrane $(43,44)$, and it reduced ureteric branching in embryonic kidney in vitro. The matrix metalloproteinase inhibitor ilomastat recapitulated the effect of TIMP-2 on both the branching of the bud and the deposition of its matrix. Thus, as with TIMP-1 (31), it appears likely that TIMP-2 regulates the morphogenesis of the ureteric bud by inhibiting matrix metalloproteinases and changing matrix composition at sites where it accumulates. This conclusion is concordant with experiments showing that the embryonic development of many epithelial tubules is modified by TIMP inhibition of matrix metalloproteinases (16, 31, 40-42).

In sum, our data suggest that factors essential for ureteric development (such as GDNF and FGF-7) not only enhance mesenchymal growth but regulate ureteric morphogenesis, in part, by stimulating the secretion of TIMP-2. That receptors for GDNF and FGF-7 (35) are located in different parts of the ureteric bud suggests that regional control of TIMP-2's secretion may determine the sites of action of this branching inhibitor and mesenchymal growth factor.

\section{Acknowledgments}

This work was supported by National Institutes of Health (NIH) grant 5PO1 DK-46934 (to J. Barasch), an American Heart Association Established Investigatorship (to J. Barasch), National Science Foundation grant DBI-9420123 (to P. Tempst), and National Cancer Institute grant 5 P30 CA-08748 (to the Sloan-Kettering Structural Chemistry Lab). J. Barasch gratefully acknowledges the generous support of the Zambetti Foundation. The authors wish to thank Theresa Swayne of the Confocal Microscopy Facility of the Herbert Irving Cancer Center at Columbia University, which was established by NIH Shared Instrument Grant 1 S10 RR-10506 and is supported by NIH grant 5 P30 CA-13696. The authors also thank David Williams for electron microscopy, Mary Lui and Lynne Lacomis for expert assistance with protein structural analysis, and Matthias Mann for use of the PeptideSearch algorithm.

1. Kreidberg, J.A., et al. 1993. WT-1 is required for early kidney development. Cell. 74:679-691.

2. Sanchez, M., et al. 1996. Renal agenesis and the absence of enteric neurons in mice lacking GDNF. Nature. 382:70-73.

3. Pichel, J., et al. 1996. Defects in enteric innervation and kidney development in mice lacking GDNF. Nature. 382:73-76.

4. Moore, M., et al. 1996. Renal and neuronal abnormalities in mice lacking GDNF Nature. 382:76-79.

5. Schuchardt, A., et al. 1994. Defects in the kidney and the enteric nervous system of mice lacking the tyrosine kinase receptor ret. Nature. 367:380-383.

6. Koseki, C., Herzlinger, D., and Al-Awqati, Q. 1992. Apoptosis in metanephric development J. Cell Biol. 119:1327-1333.

7. Coles, H.S., Burne, J.F., and Raff, M.C. 1993. Large scale neuronal cell death in the developing rat kidney and its reduction by epidermal growth factor. Development. 118:777-784.

8. Barasch, J., Pressler, L., Connor, J. and Malik, A. 1996. A ureteric bud cell line induces nephrogenesis in two steps by two distinct signals. Am.J. Physiol. 271:F50-F61.

9. Saxen, L. Organogenesis of the kidney. 1987. Cambridge University Press. Cambridge, United Kingdom. 88-128.

10. Ekblom, P. 1989. Developmentally regulated conversion of mesenchyme to epithelium. FASEB J. 3:2141-2150.

11. Vukicevic, S., Kopp, J.B., Luyten, F.P., and Sampath, T.K. 1996. Induction of nephrogenic mesenchyme by osteogenic protein-1. Proc. Natl. Acad. Sci. USA. 93:9021-9026.

12. Woolf, A., et al. 1995. Roles of hepatocyte growth factor/ scatter factor and the met receptor in the early development of the metanephros. J. Cell Biol. 128:171-184.

13. Sonnenberg, E., Meyer, D., Weidner, M., and Birchmeier, C. 1993. Scatter factor/hepatocyte growth factor and its receptor, the c-met tyrosine kinase, can mediate a signal exchange between mesenchyme and epithelia during mouse development J. Cell Biol. 123:223-235.

14. Barasch, J., et al. 1997. Ureteric bud cells secrete multiple factors, including bFGF, which rescue renal progenitors from apoptosis. Am. J. Physiol. 273:F757-F767.

15. Galardy, R.E., et al. 1994. Low molecular weight inhibitors in corneal ulceration. Ann. NY Acad. Sci. 732:315-323.

16. Chin, J., and Werb, Z. 1997. Matrix metalloproteinases regulate morphogenesis, migration and remodeling of epithelium tongue and skeletal muscle and cartilage in mandibular arch. Development. 124:1519-1530.

17. Behrendtsen, O., and Werb, Z. 1997. Metalloproteinases regulate parietal endoderm differentiating and migrating in cultured mouse embryos. Dev. Dyn. 208:255-265.

18. Murphy, G., and Crabbe, T. 1995. Gelatinases A and B. Methods Enzymol. 248:470-484.

19. Lui, M., Tempst, P., and Erdjument-Bromage, H. 1996. Methodical analysis of protein-nitrocellulose interactions to design a refined digestion protocol. Anal. Biochem. 241:156-166.

20. Elicone, C., Lui, M., Geromanos, S., Erdjument-Bromage, H., and Tempst, P. 1994. Microbore reversed phase high performance liquid chromatographic purification of peptides for combined chemical sequencing-laser desorption mass spectrometric analysis. J. Chromatogr. 676:121-137.

21. Erdjument-Bromage, H., Lui, M., Sabatini, D.M., Snyder, S.H., and Tempst, P. 1994. High sensitivity sequencing of large proteins: partial structure of the rapamycin-FKBP12 target. Protein Sci. 3:2435-2446.

22. Tempst, P., Geromanos, S., Elicone, C., and Erdjument-Bromage, H. 1994. Improvements in microsequencer performance for low picomole sequence analysis. Methods Enzymol. 6:248-261.

23. Altschul, S.F., Gish, W., Miller, W., Meyers, E.W., and Lipman, D.J. 1990. 
Basic local alignment search tool. J. Mol. Biol. 215:403-410.

24. Oliver, G.W., Leferson, J.D., Stetler-Stevenson, W.G., and Kleiner, D.E. 1997. Quantitative reverse zymography: analysis of picogram amounts of metalloproteinase inhibitors using gelatinase A and B reverse zymograms Anal. Biochem. 244:161-166.

25. Hayakawa, T., Yamashita, K., Ohuchi, E., and Shinagawa, A. 1994. Cell growth promoting activity of TIMP-2. J. Cell Sci. 107:2373-2379.

26. Means, G.E., and Feeney, R.E. 1971. Chemical modification of proteins. Holden-Day. San Francisco, CA. 214.

27. Stetler-Stevenson, W., Bersch, N., and Golde, D.W. 1992. TIMP-2 has erythroid potentiating activity. FEBS Lett. 296:231-234.

28. Nemeth, J.A., Rafe, A., Steiner, M., and Goolsby, C.L. 1996. TIMP-2 growth stimulatory activity: a concentration and cell type specific response in the presence of insulin. Exp. Cell. Res 224:110-115.

29. Corcoran, M., and Stetler-Stevenson, W. 1995. Tissue inhibitor of metalloproteinase-2 stimulates fibroblast proliferation via a cAMP-dependent mechanism. J. Biol. Chem. 270:13453-13459.

30. Guedez, L., et al. 1998. In vitro suppression of programmed cell death of B cells by tissue inhibitor of metalloproteinases-1. J. Clin. Invest. 102:2002-2010

31. Lelongt, B., Trugnan, G., Murphy, G., and Ronco, P. 1997. Matrix metalloproteinases MMP2 and MMP9 are produced in early stages of kidney morphogenesis but only MMP9 is required for renal organogenesis in vitro. J. Cell Biol. 136:1363-1373.

32. Ota, K., et al. 1998. Cloning of murine membrane type-1-matrix metalloproteinase (MT-1-MMP) and its metanephric developmental regulation with respect to MMP-2 and its inhibitor. Kidney Int. 54:131-142.

33. Vanio, S., Jalkanen, M., Bernfield, M., and Saxen, L. 1990. Transient expression of syndecan in mesenchymal cell aggregates of the embryonic kidney Dev. Biol. 152:221-232.

34. Suvanto, P., et al. 1996. Localization of glial cell derived neurotrophic factor (GDNF) mRNA in embryonic rat by in situ hybridization. Eur. J. Neurosci. 8:816-822.

35. Finch, P.W., Cuhna, G., Rubin, J.S., Wong, J., and Ron, D. 1995. Pattern of keratinocyte growth factor and keratinocyte growth factor receptor during fetal development suggests a role in mediating morphogenetic mesenchymal-epithelial interactions. Dev. Dyn. 203:223-240.

36. Treanor, J.J., et al. 1996. Characterization of a multicomponent receptor for GDNF. Nature. 382:80-83

37. Vega, Q., Worby, C.A., Lechner, M.S., Dixon, J.E., and Dressler, G.R. 1996. Glial cell line derived neurotrophic factor activates the receptor tyrosine kinase ret and promotes kidney morphogenesis. Proc. Natl. Acad. Sci. USA. 93:10656-10661.

38. Miki, T., et al. 1992. Determination of ligand-binding specificity by alternative splicing: two distinct growth factor receptors encoded by a single gene. Proc. Natl. Acad. Sci. USA. 89:249-250.

39. Nguyen, H.Q., et al. 1996. Expression of keratinocyte growth factor in embryonic liver of transgenic mice causes changes in epithelial growth and differentiation, resulting in polycystic kidneys and other organ malformations. Oncogene. 12:2109-2119.

40. Talhouk, R., Bissell, M.J., and Werb, Z. 1992. Coordinate expression of extracellular matrix degrading proteinases and their inhibitors regulates mammary epithelial function during evolution. J. Cell Biol. 118:1271-1282.

41. Witty, J.P., Wright, J.H., and Matrisian, L. 1995. Matrix metalloproteinases are expressed during ductal and alveolar mammary morphogenesis, and misregulation of stromelysin- 1 in transgenic mice induces unscheduled alveolar development. Mol. Biol. Cell. 6:1287-1303.

42. Hayakawa, T., Kishi, J., and Nakanishi, Y. 1992. Salivary gland morphogenesis: possible involvement of collagenases. Matrix Suppl. 1:344-351.

43. Ekblom, P., Alitalo, K., Vaheri, A., Timpl, R., and Saxen, L. 1980. Induction of basement membrane glycoprotein in embryonic kidney: possible role of laminin in morphogenesis. Proc. Natl. Acad. Sci. USA. 77:485-489.

44. Ekblom, P., Lehtonen, E., Saxen, L., and Timpl, R. 1981. Shift in collagen type as an early response to induction. J. Cell Biol. 89:276-283.

45. Alexander, C., Howard, E., Bissell, M., and Werb, Z. 1996. Rescue of mammary epithelial cell apoptosis and entactin degradation by a tissue inhibitor of metalloproteinase-1 transgene J. Cell Biol. 135:1669-1677.

46. Sainio, K, et al. 1997. Glial cell line derived neurotrophic factor is required for bud initiation from ureteric epithelium. Development. 124:4077-4087.

47. Davies, J.A., and Bard, J.B. 1996. Inductive interactions between the mesenchyme and the ureteric bud. Exp. Nephrol. 4:77-85.

48. Wary, K.K., et al. 1996. The adaptor protein Shc couples a class of integrins to the control of cell cycle progression. Cell. 87:733-743.

49. Levi, E., et al. 1996. Matrix metalloproteinase-2 releases active soluble ectodomain of fibroblast growth factor receptor-1. Proc. Natl. Acad. Sci. USA. 93:7069-7074.

50. Bertaux, B., Hornebeck, W., Eisen, A., and Dubertret, L. 1991. Growth stimulation of human keratinocytes by tissue inhibitor of metalloproteinases. J. Invest. Dermatol. 97:679-685.

51. Yamashita, K., et al. 1996. Tyrosine phosphorylation is crucial for growth signaling by tissue inhibitors of metalloproteinases. FEBS Lett. 396:103-107.

52. Chesler, L., Golde, D.W., Bersch, N., and Johnson, M. 1995. Metalloproteinase inhibition and erythroid potentiation are independent activities of tissue inhibitor of metalloproteinases-1. Blood. 86:4506-4515.

53. Laufer, E., Nelson, C.E., Johnson, R.L., Morgan, B.A., and Tabin, C. 1994. Sonic hedgehog and FGF-4 act through a signaling cascade and feedback loop to integrate growth and patterning of the developing limb bud. Cell. 79:993-1003.

54. Niswander, L., Jeffrey, S., Martin, G.R., and Tickle, C. 1994. A positive feedback loop coordinates growth and patterning in the vertebrate limb. Nature. 371:609-612.

55. Kispert, A., Vainio, S., Shen, L., Rowitch, D.H. and McMahon, A.P. 1996. Proteoglycans are required for maintenance of Wnt-11 expression in the ureter tips. Development. 122:3627-3637. 